\title{
The numerical analysis of the interaction between the low-order Lamb wave modes and surface breaking cracks
}

\author{
Michal Šofer ${ }^{1, *}$, Petr Ferfecki ${ }^{1,2}$, Martin Fusek ${ }^{1}$, Pavel Šofer $^{3}$, Renata Gnatowska ${ }^{4}$ \\ ${ }^{1}$ Department of Applied Mechanics, Faculty of Mechanical Engineering, VŠB-Technical University of \\ Ostrava, 17. listopadu 15/2127, 70833 Ostrava-Poruba, Czech Republic \\ ${ }^{2}$ IT4Innovations National Supercomputing Center, VŠB-Technical University of Ostrava, \\ 17. listopadu 15/2127, 70833 Ostrava-Poruba, Czech Republic \\ ${ }^{3}$ Department of Control Systems and Instrumentation, Faculty of Mechanical Engineering, \\ VŠB-Technical University of Ostrava, 17. listopadu 15/2127, 70833 Ostrava-Poruba, Czech Republic \\ ${ }^{4}$ Department of Control Systems and Instrumentation, Faculty of Mechanical Engineering and \\ Computer Science, Institute of Thermal Machinery, Czestochowa University of Technology, Armii \\ Krajowej 21, 42-200 Czestochowa, Poland
}

\begin{abstract}
Lamb waves, as one of the types of guided waves, are extensively used for inspecting large structures as well as for structure health monitoring applications. One of the biggest benefits of guided waves is their ability to travel over long distances without much attenuation. Lamb waves are often used for inspection of piping systems and similar geometries where the dimension in the third direction is significantly smaller than the other two. No wonder that the study of the interaction of Lamb waves with particular types of geometric discontinuities is a frequent topic of research. The main aim of the proposed paper is to present the findings related to the numerical study of the interaction between low-order Lamb wave modes and surface breaking crack oriented at different angles relative to the free surface.
\end{abstract}

Keywords: Lamb wave, Surface breaking crack, Normal mode theory, Orthogonality relation

\section{Introduction}

Lamb waves are extensively used for inspecting large structures as well as for structure health monitoring (SHM) [1]. The main feature of Lamb waves is that they can exist only in geometries with a finite thickness such as plates, rods, or tubes [2]. Lamb waves have the property of being able to travel over long distances without much attenuation; therefore, an interaction of Lamb waves with particular types of geometric discontinuities has been an important topic of research in terms of development of experimental, numerical as well as semi-analytical approaches [3-6]. Particularly in the case of experimental methods, the

\footnotetext{
* Corresponding author: michal.sofer@,vsb.cz

Reviewers: Czestaw Kundera, Tomáš Lack
} 
retrospective characterization of the present defect with regard to the dispersive nature of Lamb waves present a relatively complicated matter. The increasing number of Lamb modes in the structure helps us to increase the informative level of the eventually present discontinuity, but, at the same time, the analysis of experimental data in itself is more difficult $[4,7]$. In order to be able to solve such inverse problem, i.e. the backward characterization of the defect based on the measured experimental data, it is necessary to utilize methods that allow the interaction of selected Lamb wave modes with particular types of discontinuities to be accurately predicted. For these purposes, it is possible to use approaches based on the incorporation of the finite element method [8], boundary element method [9], or available alternatives in the form of hybrid methods, such as the Semi Analytical Finite Element Method (SAFEM) [10], which is based on the discretization of the cross-section through the finite elements and subsequent use of the classical analytical solution for wave propagation in the chosen direction. The advantage of this method is the possibility to solve the wave propagation in waveguides with geometrically complicated cross-section including propagation in composite plates.

An equally important group of methods are hybrid methods using a combination of finite or boundary element method and analytical approach, which is most often based on the principle of modal expansion and the orthogonal property of Lamb wave modes [11]. A number of authors, however, have focused their efforts on the development of fully analytical models that would completely eliminate the utilization of the finite or boundary element method as the tool for determination of stress and displacement field in the vicinity of the discontinuity $[1,6]$.

The main aim of the proposed paper is to perform a numerical study, which is focused on an interaction between the low-order Lamb wave modes and surface breaking crack with a constant projected depth, oriented in a wide range of angles relative to the free surface. The numerical analysis of the energy transmission and reflection coefficients identification is performed using a semi-analytical method [11], which incorporates both the normal mode expansion theory and the orthogonal property of Lamb wave modes. The input data are then the through-thickness displacement vector and stress tensor components, which are obtained using the finite element method (FEM) in front of and behind the discontinuity.

\section{Semi-Analytical method for energy scattering coefficients evaluation}

The semi-analytical model utilized for numerical study was published in 2006 by L. Moreau, M. Castaings, and B. Hosten [11]. The basis of the model is incorporation of the modal expansion technique and the orthogonal property of Lamb waves. Lamb modes are the solutions of the elasticity equation in case of uniform waveguide with free boundaries on both sides of the waveguide:

$$
\mu \nabla^{2} \mathbf{u}+(\lambda+\mu) \nabla(\operatorname{div} \mathbf{u})=\rho \ddot{\mathbf{u}},
$$

where $\lambda$ and $\mu$ denotes Lame constants, $\rho$ is density of the material, and $\ddot{\mathbf{u}}$ denotes second time $t$ derivate of displacement vector $\mathbf{u}$.

The solution forms a discrete spectrum of wavenumbers, which can be split into two parts according to the wave propagation direction (positive vs negative $x$ axis) [6]. The important fact is that the individual Lamb modes, which can be treated as eigenfunctions, form a complete orthogonal basis [12]. Due to these assumptions, it is possible to apply the eigenfunctions expansion technique on the displacement vector $\mathbf{u}_{\alpha}^{n}$ and the stress tensor $\overline{\overline{\boldsymbol{\sigma}}}_{\alpha}^{n}$ of the $n$-th Lamb wave mode: 


$$
\begin{gathered}
\mathbf{u}_{\alpha}^{n}(x, z)=\alpha_{n}(x) \mathbf{u}^{n}(z)=\alpha_{n}(x)\left(\begin{array}{l}
u^{n}(z) \\
v^{n}(z)
\end{array}\right), \\
\overline{\overline{\mathbf{\sigma}}}_{\alpha}^{n}(x, z)=\alpha_{n}(x) \overline{\overline{\boldsymbol{\sigma}}}^{n}(z)=\alpha_{n}(x)\left[\begin{array}{cc}
\sigma_{x}^{n}(z) & \tau_{x z}^{n}(z) \\
\tau_{x z}^{n}(z) & \sigma_{z}^{n}(z)
\end{array}\right],
\end{gathered}
$$

where: $\alpha_{n}$ is the complex amplitude related to $\mathrm{n}$-th mode, $\mathbf{u}^{n}(z)$ and $\overline{\overline{\boldsymbol{\sigma}}}^{n}(z)$ are the displacement vector and the stress tensor, respectively, which are a function of the $z$ coordinate and $u^{n}(z), v^{n}(z), \sigma_{x}^{n}(z), \sigma_{z}^{n}(z)$, and $\tau_{x z}^{n}(z)$ are the displacement vector and stress tensor components. It has to be also noted, that both the displacement vector and the stress tensor are a function of $e^{i\left(\omega t-k_{n} x\right)}$ term, where $k_{n}$ is the wavenumber of n-th Lamb wave mode and $\omega$ is angular frequency.

The time-averaged power flow $P_{A V x}^{n}$ for the n-th mode, which propagates in guide with thickness $t_{h}$, can be expressed as follows:

$$
P_{A V x}^{n}=\operatorname{Re}\left[\int_{0}^{t_{h}}-\overline{\overline{\mathbf{\sigma}}}_{\alpha}^{n} \dot{\mathbf{u}}_{\alpha}^{n *} \boldsymbol{i} \mathrm{d} z\right],
$$

where: $\boldsymbol{i}$ denotes unit vector in the $x$ direction, the ${ }^{*}$ denotes complex conjugation, the ${ }^{\bullet}$ denotes time derivative and $t_{h}$ represents plate thickness for which apply: $t_{h}=2 h$ (see Figure 1). After some manipulation and taking into account the assumption of plane strain condition equation (4) can be rewritten in the following form:

$$
P_{A V x}^{n}=\alpha_{n} \alpha_{n}{ }^{*} \operatorname{Re}\left[i \omega \int_{0}^{t_{h}}\left(\sigma_{x}^{n} u^{n^{*}}+\tau_{x z}^{n} v^{n *}\right) \mathrm{d} z\right] .
$$

According to the form of $P_{A V x}^{n}$, it would be favourable to normalize the stress tensor and displacement vector components in order to express the power flow solely in terms of the complex amplitude $\alpha_{n}$ [11]. Let us define the stress tensor and displacement vector in modified form:

$$
\begin{gathered}
\widetilde{\mathbf{u}}_{\alpha}^{n}(x, z)=\alpha_{n}(x) \widetilde{\mathbf{u}}^{n}(z)=\alpha_{n}(x)\left(\begin{array}{c}
\tilde{u}^{n}(z) \\
\tilde{v}^{n}(z)
\end{array}\right), \\
\overline{\overline{\boldsymbol{\sigma}}}_{\alpha}^{n}(x, z)=\alpha_{n}(x) \overline{\overline{\widetilde{\sigma}}}^{n}(z)=\alpha_{n}(x)\left[\begin{array}{cc}
\tilde{\sigma}_{x}^{n}(z) & \tilde{\tau}_{x z}^{n}(z) \\
\tilde{\tau}_{x z}^{n}(z) & \tilde{\sigma}_{z}^{n}(z)
\end{array}\right],
\end{gathered}
$$

where symbol $\sim$ expresses the normalization with use of $\frac{1}{\sqrt{\left|P_{A V x}^{n}\right|}}$ term. After repeated substitution of Equation (6) and (7) into Equation (5), we will obtain a modified expression for power flow, which will be now solely dependent on the value of complex amplitude $\alpha_{n}$ :

$$
P_{A V x}^{n}=\alpha_{n} \alpha_{n}^{*}=\left|\alpha_{n}\right|^{2} .
$$

Suppose that the total wave field can be expressed as a linear combination of all, for a given $f t_{h}$ parameter, existing Lamb wave modes where $f$ is a frequency and $t_{h}$ is plate thickness:

$$
\mathbf{u}_{S U M}(x, z)=\sum_{N} \alpha_{n}(x) \widetilde{\mathbf{u}}^{n}(z)
$$




$$
\boldsymbol{\sigma}_{S U M}(x, z)=\sum_{N} \alpha_{n}(x) \overline{\overline{\boldsymbol{\sigma}}}^{n}(z) .
$$

One of the most important properties of Lamb waves is the orthogonality between two different modes. In literature, several orthogonality conditions that differ from one another to their intended use can be found. In this case, we will use the orthogonality condition published by Shkerdin and Glorieux [5]:

$$
\int_{0}^{t_{h}}\left(\tilde{\sigma}_{x}^{n} \tilde{u}^{m}+\tilde{\sigma}_{x}^{m} \tilde{u}^{n}-\tilde{\tau}_{x z}^{n} \tilde{v}^{m}-\tilde{\tau}_{x z}^{m} \tilde{v}^{n}\right) d z=\Lambda_{m n} \delta_{m n}
$$

where $\delta_{m n}$ is Kronecker symbol and $\Lambda_{m n}$ is the result of the integral for $m \neq n$, which is subsequently used for calculation of complex amplitude of the given mode. Based on the known through-thickness displacement and stress components, it is possible to rewrite Equation (11) into following form:

$$
\int_{0}^{t_{h}}\left(\tilde{\sigma}_{x}^{n} u_{S U M}+\sigma_{x S U M} \tilde{u}^{n}-\tilde{\tau}_{x z}^{n} v_{S U M}-\tau_{x z S U M} \tilde{v}^{n}\right) d z=\Lambda_{n}
$$

from where we can calculate the scalar value of the integral. Individual components $u_{S U M}$, $\sigma_{x S U M}, v_{S U M}, \tau_{x Z \text { SUM }}$ can be further replaced by Equations (9) and (10) in order to get a modified version of Equation (12):

$$
\int_{0}^{t_{h}}\left[\tilde{\sigma}_{x}^{n} \sum_{N} \alpha_{n} \tilde{u}^{n}+\sum_{N} \alpha_{n} \tilde{\sigma}_{x}^{n} \tilde{u}^{n}-\tilde{\tau}_{x z}^{n} \sum_{N} \alpha_{n} \tilde{v}^{n}-\sum_{N} \alpha_{n} \tilde{\tau}_{x z}^{n} \tilde{v}^{n}\right] d z=\Lambda_{n} .
$$

From Eq. (13) the relation for complex amplitude can finally be derived:

$$
\alpha_{n}=\Lambda_{n}\left\{2 \int_{0}^{t_{h}}\left[\tilde{\sigma}_{x}^{n} \tilde{u}^{n}-\tilde{\tau}_{x z}^{n} \tilde{v}^{n}\right] d z\right\}^{-1}
$$

After identification of complex amplitudes of incident, reflected and transmitted modes, it is possible to calculate the energy scattering coefficients according to the following relations:

$$
\begin{gathered}
k_{\text {ref }}^{n}=\frac{P_{A V \text { REF }}^{n}}{P_{A V I N}}=\frac{\left|\alpha_{n \text { REF }}\right|^{2}}{\left|\alpha_{n I N}\right|^{2}}, \\
k_{\text {trans }}^{n}=\frac{P_{A V T R A N S}^{n}}{P_{A V I N}}=\frac{\left|\alpha_{n T R A N S}\right|^{2}}{\left|\alpha_{n I N}\right|^{2}},
\end{gathered}
$$

where $P_{A V I N}^{n}, P_{A V R E F}^{n}, P_{A V T R A N S}^{n}$ is the power flow of incident, reflected, and transmitted n-th Lamb mode with corresponding complex amplitude $\alpha_{n I N}, \alpha_{n R E F}$, and $\alpha_{n \text { TRANS }}$.

\section{Numerical parametric study}

The finite element simulations were realized in the frequency domain in order to obtain the above-mentioned through-thickness displacements and stress distribution in front of and above the discontinuity. The subject of interest was a $3 \mathrm{~mm}$ thick aluminium plate (Young modulus $E=69 \mathrm{GPa}$, Poisson's ratio $v=0.34$, density $\rho=2660 \mathrm{~kg} / \mathrm{m}^{3}$ ), which contains a $0.1 \mathrm{~mm}$ thick surface breaking crack with the same value of crack root rounding. The crack orientation has been defined by inclination angle $\beta$ - see Figure 1, which varied within -70 
and 70 degrees. The each parametric study has been performed with a 0.5 degree step. A total of nine parametric studies were performed and differed in the excitation frequency of the incident A0 Lamb wave mode as well as in the projected depth $H$ of the surface breaking crack. For clarity, all realized variants of the parametric studies are listed in Table 1.

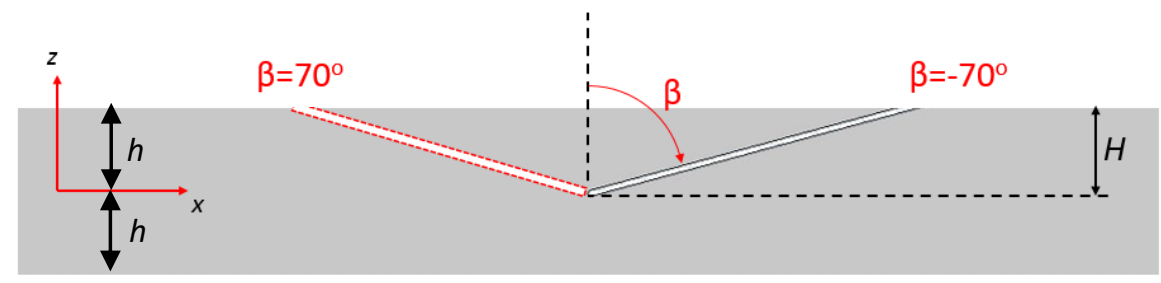

Fig. 1. Free plate including the surface breaking crack

Table 1. Realized variants of the parametric studies

\begin{tabular}{|c|c|c|c|c|}
\hline $\begin{array}{c}\text { Parametric } \\
\text { study No. } \\
{[-]}\end{array}$ & $\begin{array}{c}\text { Projected depth } H \text { in } \\
\text { percent of the plate } \\
\text { thickness } \\
{[\%]}\end{array}$ & $\begin{array}{c}\text { Excitation frequency } \\
\text { of incident A0 mode } \\
{[\mathrm{kHz}]}\end{array}$ & $\begin{array}{c}\text { Range of angle } \\
\beta \\
{\left[{ }^{\circ}\right]}\end{array}$ & $\begin{array}{c}\text { Angle step } \\
{\left[{ }^{\circ}\right]}\end{array}$ \\
\hline 1 & 25 & 100 & -70 to 70 & 0.5 \\
\hline 2 & 50 & 100 & -70 to 70 & 0.5 \\
\hline 3 & 75 & 100 & -70 to 70 & 0.5 \\
\hline 4 & 50 & 200 & $-70 \div 70$ & 0.5 \\
\hline 5 & 50 & 300 & $-70 \div 70$ & 0.5 \\
\hline 6 & 50 & 400 & $-70 \div 70$ & 0.5 \\
\hline 7 & 25 & 500 & $-70 \div 70$ & 0.5 \\
\hline 8 & 50 & 500 & $-70 \div 70$ & 0.5 \\
\hline 9 & 75 & 500 & $-70 \div 70$ & 0.5 \\
\hline
\end{tabular}

The generation of the incident mode has been performed using locally applied excitation of normal force $F(x, f)$ with a Gaussian-windowed spatial distribution by the following function [11]:

$$
F(x, f)=F_{0} \mathrm{e}^{-i k x} \mathrm{e}^{\frac{A\left(x-x_{0}\right)^{2}}{B^{2}}},
$$

where: $F_{0}$ (set at $100 \mathrm{~N}$ ) represents the amplitude of the excitation force, $k$ is the wavenumber, $x$ refers to the spatial coordinate, $A$ (set at $30 \mathrm{~mm}$ ) defines the width of the excitation zone, $B$ (set at $60 \mathrm{~mm}$ ) defines its length and $x_{0}$ is the centre of the excitation interval for which apply: $x_{0}=0.5\left(x_{2}-x_{1}\right)-$ see Figure 2 . The wavenumber of the incident A0 mode is a function of material properties, thickness of the plate as well as a function of the wave frequency. Figure 2 displays the scheme of the finite element model including the places where the through-thickness displacements and stress components were monitored.

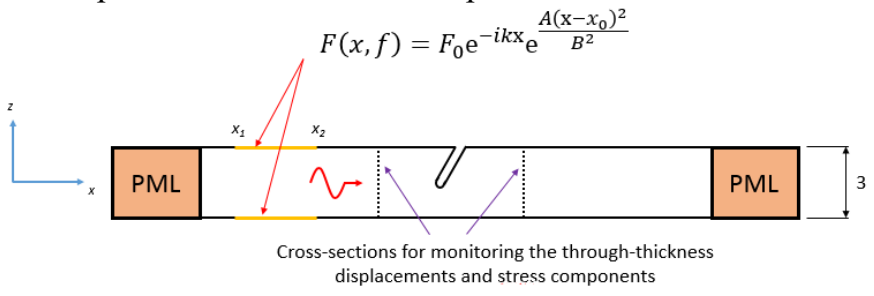

Fig. 2. Scheme of the finite element model for obtaining through-thickness displacements and stress components 
The PML abbreviation expresses the Perfectly Matched Layer property, which is an artificial absorbing layer option available in the COMSOL software. The PML property enables us to minimize the dimensions of the model including the elimination of the backwall reflection at the same time. The results from each computed parametric study were then post-processed in order to extract the required through-thickness displacements and stress components at monitored cross section segments (see Figure 2). The extracted data files were subsequently imported into the Matlab software environment where a semi-analytical approach has been utilized in order to calculate the energy scattering coefficients.

\section{Results and discussion}

Figures 3 and 4 display the functional dependencies between the reflection and transmission coefficients of the A0 Lamb wave mode and inclination angle $\beta$ for the five selected excitation frequencies of the incident $\mathrm{A} 0$ mode. It has to be noted that the results displayed in Figures 3 and 4 are for the case when the crack projected depth is equal to $50 \%$ of the plate thickness. We can notice that with the increased excitation frequency there is a substantial decrease of the interval width with the A0 energy reflection coefficient lower than $20 \%$ (see Figure 3). Thus, if we use a test methodology based on the evaluation of the reflected fraction of the A0 mode, the sensitivity of this mode in the form of change of the reflected energy due to the presence of the defect will not be satisfactory, especially within the $-60^{\circ}$ to $60^{\circ}$ interval of inclination angle $\beta$ and the $f t_{h}$ value equal to $0.3 \mathrm{MHz} \cdot \mathrm{mm}$.

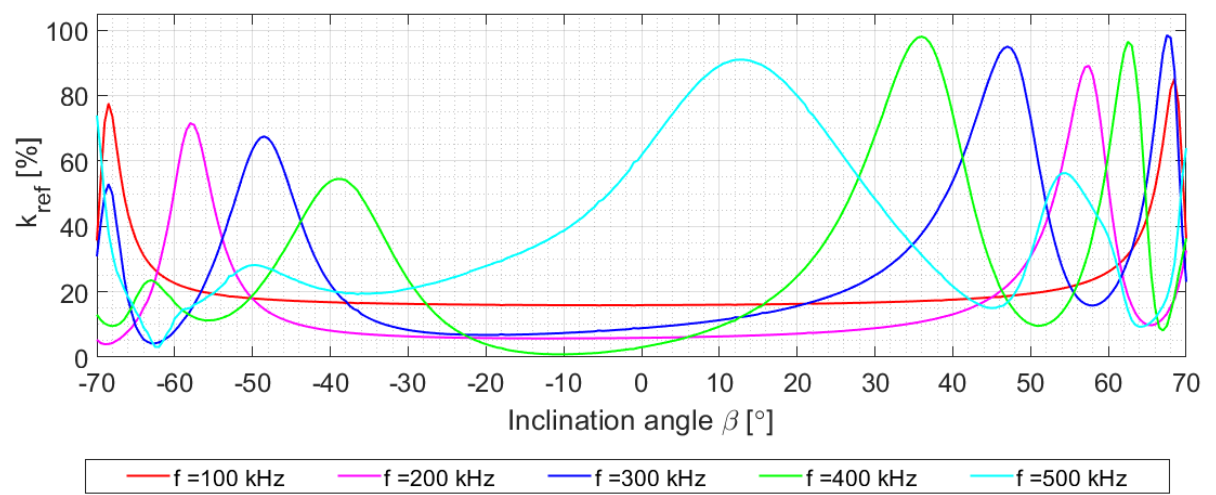

Fig. 3. Energy reflection coefficients of the A0 Lamb wave mode for given interval of inclination angle $\beta$ as the function of the excitation frequency of the incident A0 wave ( $H=50 \%$ of plate thickness)

However, with the increased $f t_{h}$ value, this interval is reduced and for $f t_{h}=1.5 \mathrm{MHz} \cdot \mathrm{mm}$ we are already achieving satisfactory results, especially in the $-10^{\circ}$ to $30^{\circ}$ angle range. Somewhat more positive results are obtained in the case of monitoring the transmitted fraction of the A0 mode (see Figure 4) where we register a very satisfying sensitivity on a relatively wide range of inclination angle $\beta$ for the lowest value of the $f t_{h}$ parameter. However, with the increasing value of the excitation frequency we are gradually losing the advantage, and the situation will improve to some extent with $f t_{h}$ parameter larger than $1.2 \mathrm{MHz} \cdot \mathrm{mm}$. A similar trend occurs when we monitor the transmitted or reflected fraction of the S0 mode (Figures 5 and 6) but with a much worse prognosis. In order to effectively cover the widest possible interval of inclination angle $\beta$ with sufficient sensitivity to detect the defect present in the given configuration, it is therefore necessary to apply combined monitoring of both, the transmitted and reflected fractions of the A0/S0 modes. For more detailed analysis of the behaviour of each of the fundamental Lamb wave modes, numerical studies with $H$ parameter equal to 
$25 \%$ and $75 \%$ have been performed. Figures 7 and 8 show the transmission and reflection coefficients of both Lamb wave modes as the function of inclination angle $\beta$ and the projected depth as the function of the $H$ parameter.

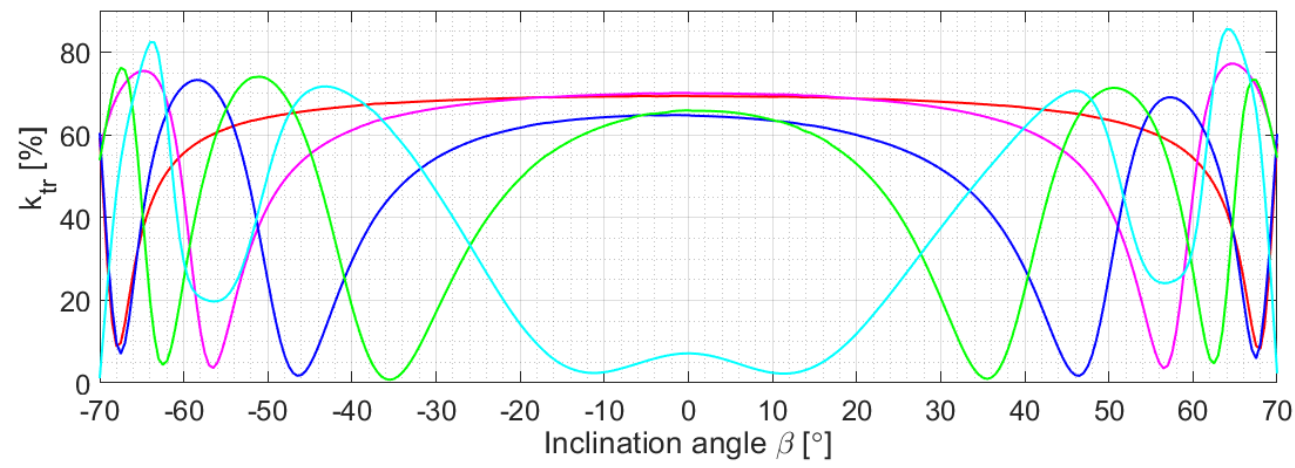

$-f=100 \mathrm{kHz}-\mathrm{f}=200 \mathrm{kHz} \quad-\mathrm{f}=300 \mathrm{kHz} \quad-\mathrm{f}=400 \mathrm{kHz} \quad-\mathrm{f}=500 \mathrm{kHz}$

Fig. 4. Energy transmission coefficients of the A0 Lamb wave mode for the given interval of inclination angle $\beta$ as the function of excitation frequency of the incident $\mathrm{A} 0$ wave ( $H=50 \%$ of plate thickness)

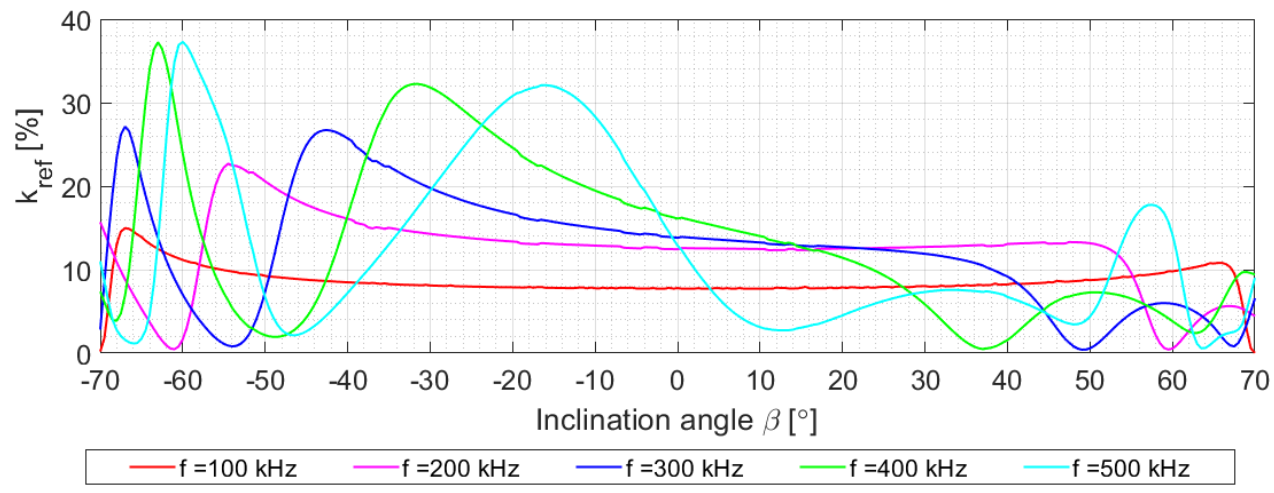

Fig. 5. Energy reflection coefficients of the $\mathrm{S} 0 \mathrm{Lamb}$ wave mode for the given interval of inclination angle $\beta$ as the function of excitation frequency of the incident A0 wave ( $H=50 \%$ of plate thickness)

The presented results show that especially for $f t=0.3 \mathrm{MHz} \cdot \mathrm{mm}$ the sensitivity of both fundamental modes to the present defect of the given configuration is very low. With the excitation frequency of $500 \mathrm{kHz}$ we will, however, obtain diametrically different results (Figures 9 and 10) in which the A0 Lamb wave mode is an advantage. In order to find out in more detail the nature of the behaviour of the individual modes, the visualization of the structure of both fundamental modes for excitation frequencies of $100 \mathrm{kHz}$ and $500 \mathrm{kHz}$ was performed - see Figures 11 and 12. The analysis shows that the rate of sensitivity related to the discontinuity detection of a given configuration is basically influenced by two main factors - the wave structure including its associated wavelength. In the case of low values of the $f_{h}$ parameter $(0,3 \mathrm{MHz} \mathrm{mm})$, we are facing diametrically different structures of the A0 and $\mathrm{S} 0$ modes. While the A0 mode with the excitation frequency $100 \mathrm{kHz}$ has a wavelength of less than 16 millimeters, including the dominant displacement in the transversal direction, the wavelength of the S0 mode at the same frequency is almost 3.5 times higher with dominant displacement in the longitudinal direction. 


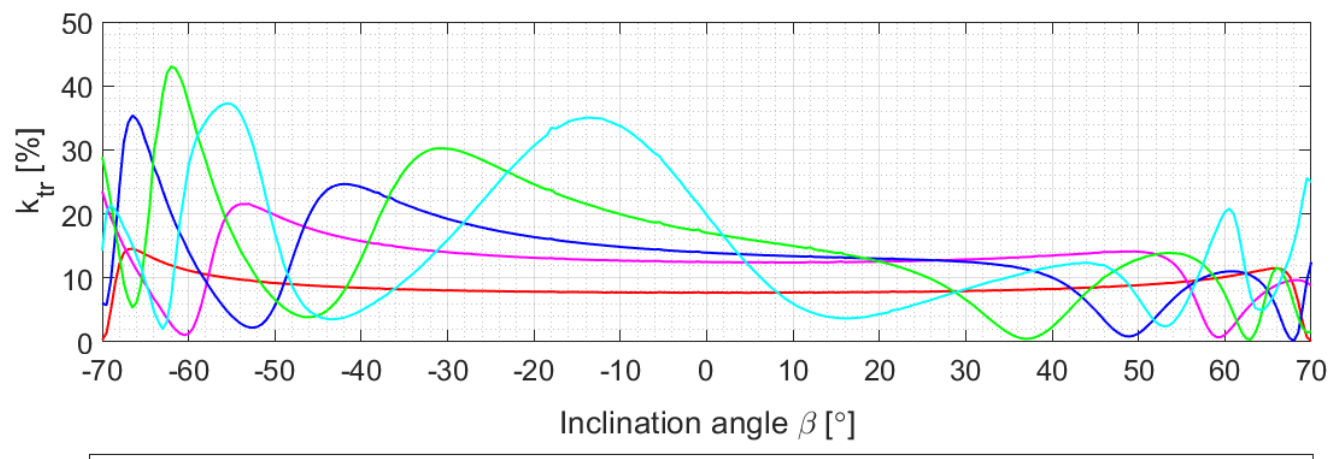

Fig. 6. Energy transmission coefficients of the S0 Lamb wave mode for the given interval of inclination angle $\beta$ as the function of the excitation frequency of incident A 0 wave ( $H=50 \%$ of plate thickness)

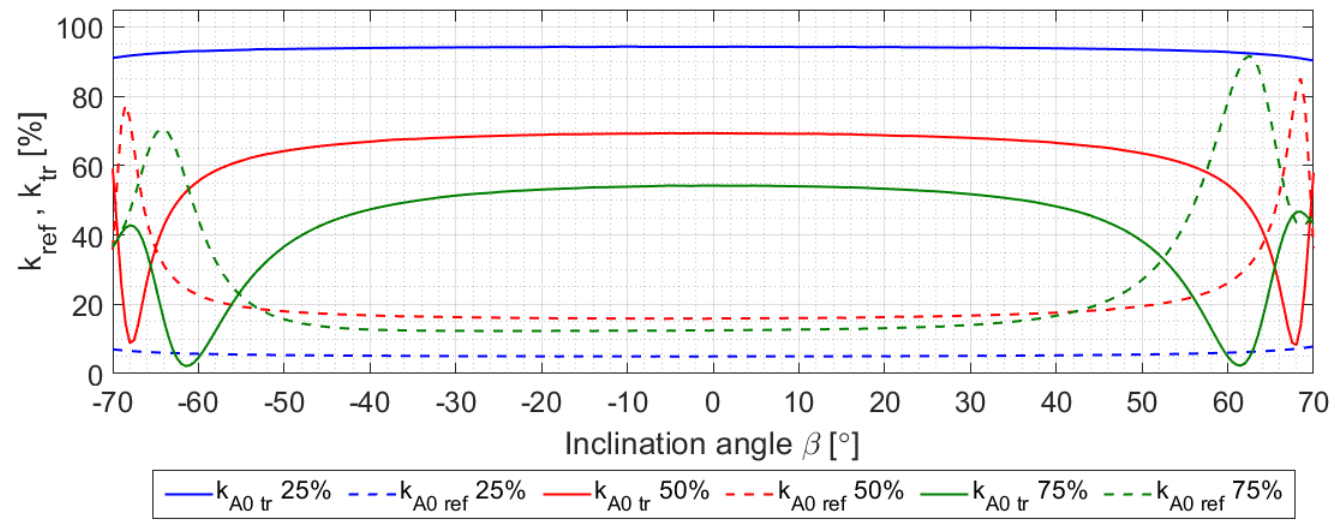

Fig. 7. Energy transmission coefficients of the $\mathrm{A} 0 \mathrm{Lamb}$ wave mode for the given interval of inclination angle $\beta$ as the function of projected crack height $H(25 \%, 50 \%$ and $75 \%$ of plate thickness). Excitation frequency of A0 mode $f=100 \mathrm{kHz}$

If we raise the frequency five times, i.e. to the value of $500 \mathrm{kHz}$, the wavelength of the S0 mode will reduce approximately five times, while the A0 wavelength is only three times lower due to a more pronounced dispersion effect. Due to much closer values of wavelengths compared to the projected dimensions of the defect in the $x$ and $z$ axes, we will register a generally higher sensitivity of both modes to the change of inclination angle $\beta$ as well as the presence of the defect itself. The presented results show, that it is important to carry out a comprehensive analysis of the individual energy scattering coefficients, including the information related to the wave structure, which are an indispensable tool for the design of the inspection methodology in the technical practice. 


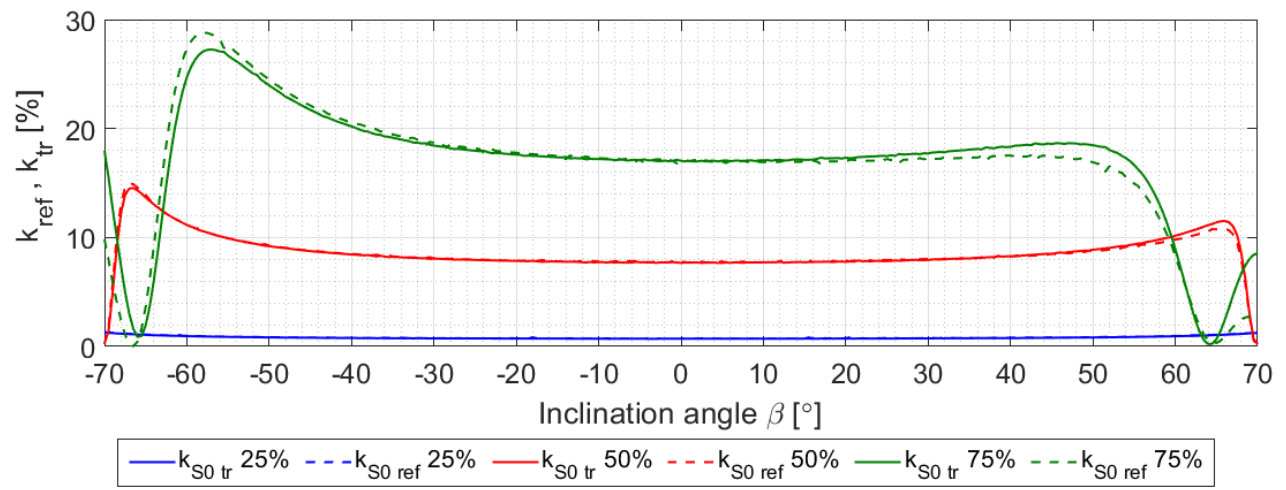

Fig. 8. Energy transmission coefficients of the S0 Lamb wave mode for the given interval of inclination angle $\beta$ as the function of projected crack height $H(25 \%, 50 \%$ and $75 \%$ of plate thickness). Excitation frequency of $\mathrm{A} 0$ mode $f=100 \mathrm{kHz}$

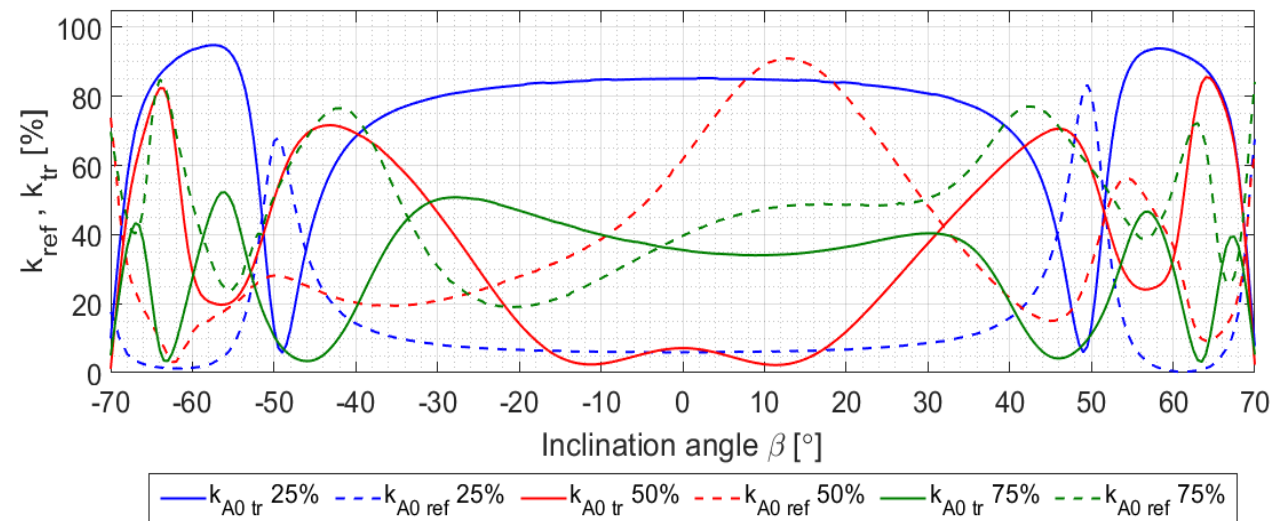

Fig. 9. Energy transmission coefficients of A0 Lamb wave mode for the given interval of inclination angle $\beta$ as the function of projected crack height $H(25,50$ and $75 \%$ of plate thickness). Excitation frequency of $\mathrm{A} 0$ mode $f=500 \mathrm{kHz}$

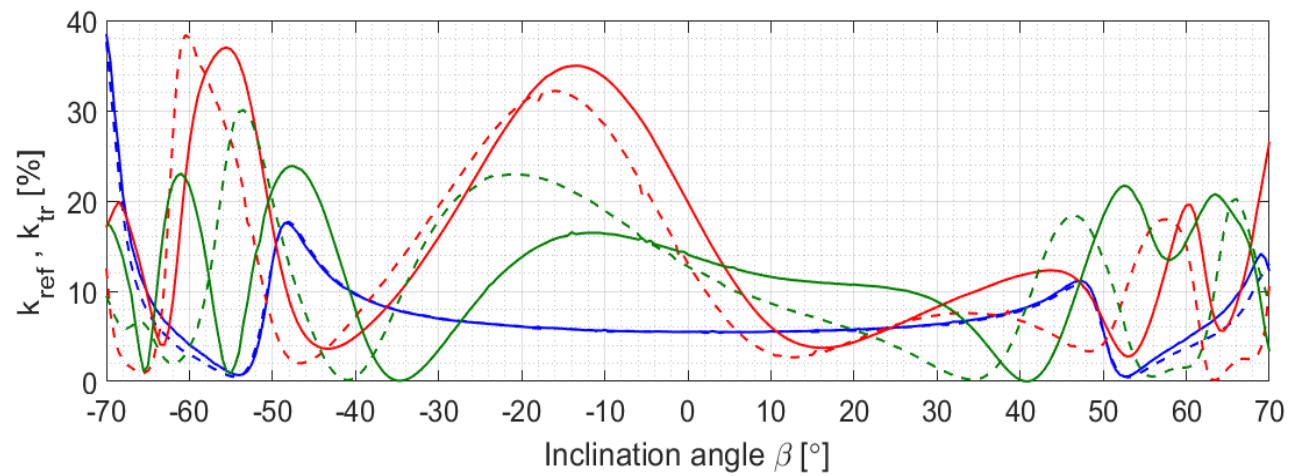

$-\mathrm{k}_{\mathrm{SO} \text { tr }} 25 \%--\mathrm{k}_{\mathrm{SO} \text { ref }} 25 \%-\mathrm{k}_{\mathrm{SO} \text { tr }} 50 \%--\mathrm{k}_{\mathrm{SO} \text { ref }} 50 \%-\mathrm{k}_{\mathrm{SO} \text { tr }} 75 \%---\mathrm{k}_{\mathrm{sO} \text { ref }} 75 \%$

Fig. 10. Energy transmission coefficients of the S0 Lamb wave mode for the given interval of inclination angle $\beta$ as the function of projected crack height $H(25 \%, 50 \%$ and $75 \%$ of plate thickness). Excitation frequency of A0 mode $f=500 \mathrm{kHz}$ 

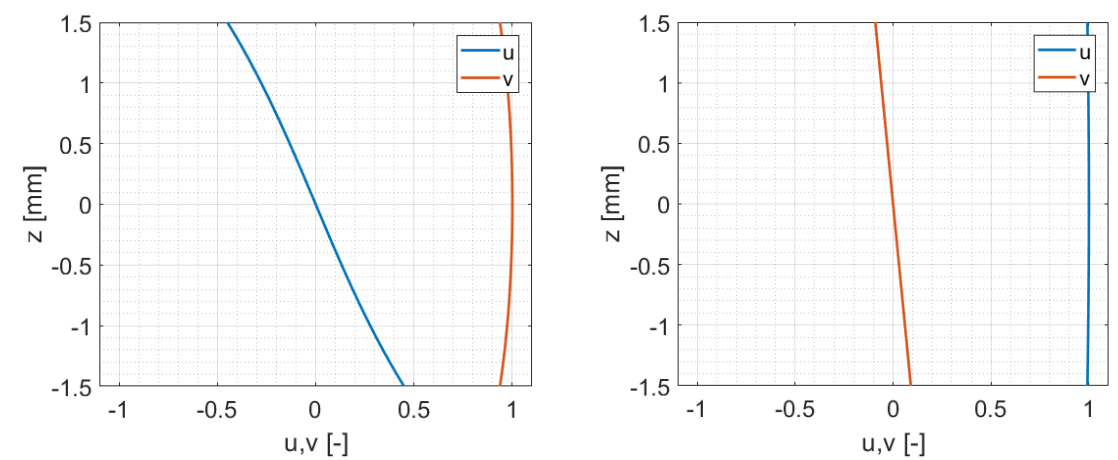

Fig. 11. Normalized displacements of $\mathrm{A} 0$ mode (left) and $\mathrm{S} 0$ mode (right), $f=100 \mathrm{kHz}, f t=0.3 \mathrm{~Hz} \cdot \mathrm{mm}$
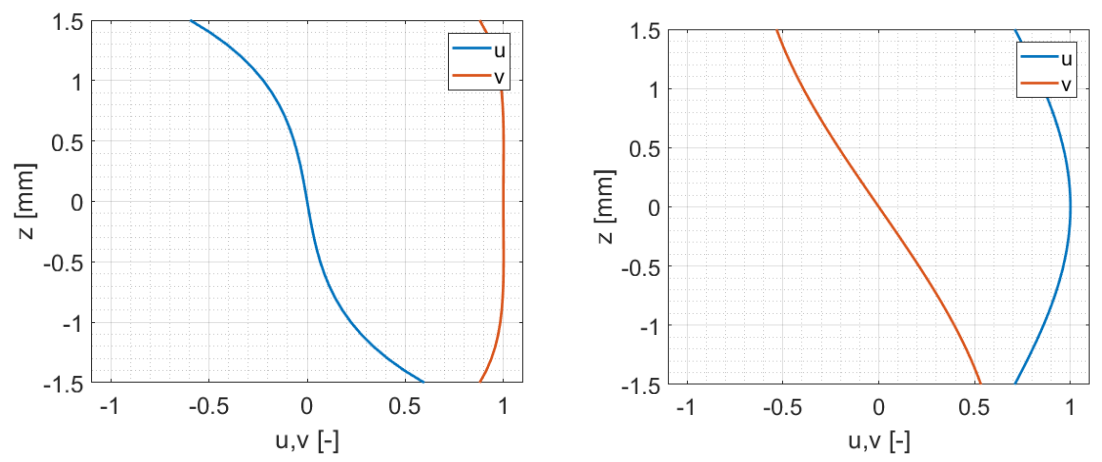

Fig. 12. Normalized displacements of $\mathrm{A} 0$ mode (left) and $\mathrm{S} 0$ mode (right), $f=500 \mathrm{kHz}$, $f t=1.5 \mathrm{MHz} \cdot \mathrm{mm}$

\section{Conclusion}

The main aim of the presented paper was to perform a detailed numerical study related to the usability of fundamental A0 and S0 Lamb wave modes for detection of specified discontinuity type, namely surface breaking cracks of different projected depths. The study has revealed completely different behaviour of A0 and S0 modes in terms of the interaction with the above-mentioned discontinuity type. It has been shown that the decisive influence on detection sensitivity basically has two aspects - the structure of the wave in terms of the ration between displacements in the $x$ and $z$ axes and the wavelength of the wave. The information obtained so far will be subsequently applied to a series of planned experimental studies with the aim to verify numerical results on the cases of real discontinuities.

This work was supported by Specific Research (SP2018/63) and by The Ministry of Education, Youth and Sports from the National Programme of Sustainability II (LQ1602). The support is acknowledged.

\section{References}

1. B. Poddar, V. Giurgiutiu. Complex Modes Expansion with Vector Projection using Power Flow to Simulate Lamb Waves Scattering from Horizontal Cracks and Disbonds. Journal of the American Chemical Society 140, 2123-2133 (2016) 
2. T.N. Grigsby, E.J. Tajchman. Properties of Lamb Waves Relevant to the Ultrasonic Inspection of Thin Plates. Transactions on Ultrasonic Engineering 8, 26-33 (1961)

3. F. Feng, J. Shen, J. Deng, Q. Wang. Analytical Solution of Lamb Wave Scattering at Plate End. Advanced Materials Research 199-200, 949-952 (2011)

4. J. L. Rose, Ultrasonic Guided Waves in Solid Media. Cambridge University Press (2014)

5. G. Shkerdin, C. Glorieux. Interaction of Lamb modes with an Inclusion. Ultrasonics 53, 130-140 (2013)

6. F. Feng, J. Shen, S. Lin. Scattering Matrices of Lamb Waves at Irregular Surface and Void Defects. Ultrasonics 52, 760-766 (2012)

7. B. Poddar. Physics Based Modeling of Guided Waves for Detection and Characterization of Structural Damage in NDE and SHM. Dissertation Thesis, University of South Carolina 187p (2016)

8. M. Koshiba, M. Morita, M. Suzuki. Finite-element analysis of discontinuity problem of SH modes in an elastic plate waveguide. Electronics Letters 17, 480-483 (1981)

9. J. Galán, R. Abascal. Lamb Mode Conversion at Edges. A Hybrid Boundary ElementFinite-Element Solution. The Journal of the Acoustical Society of America 117, 17771784 (2005)

10. T. Hayashi, W.J. Song, J.L. Rose. Guided Wave Dispersion Curves for a Bar with an Arbitrary Cross-Section, a Rod and Rail Example. Ultrasonics 41, 175-183 (2003)

11. L. Moreau, M. Castaings, B. Hosten. An orthogonality relation-based technique for postprocessing finite element predictions of waves scattering in solid waveguides. The Journal of the Acoustical Society of America 120, 611-620 (2006)

12. P. Kirrmann. On the completeness of Lamb modes. Journal of Elasticity 37, 39-69 (1994) 